\title{
Toward a New Geometric Distance To the Active Galaxy NGC 4258. III. Final Results and The Hubble Constant
}

\section{Citation}

Humphreys, E. M. L., Mark Jonathan Reid, James M. Moran, Lincoln Jared Greenhill, and Alice L. Argon. 2013. Toward A New Geometric Distance To The Active Galaxy NGC 4258. III. Final Results and The Hubble Constant. The Astrophysical Journal 775(1): 13.

\section{Published Version}

doi:10.1088/0004-637X/775/1/13

\section{Permanent link}

http://nrs.harvard.edu/urn-3:HUL.InstRepos:12561432

\section{Terms of Use}

This article was downloaded from Harvard University's DASH repository, and is made available under the terms and conditions applicable to Other Posted Material, as set forth at http:// nrs.harvard.edu/urn-3:HUL.InstRepos:dash.current.terms-of-use\#LAA

\section{Share Your Story}

The Harvard community has made this article openly available.

Please share how this access benefits you. Submit a story.

\section{Accessibility}




\title{
TOWARD A NEW GEOMETRIC DISTANCE TO THE ACTIVE GALAXY NGC 4258. III. FINAL RESULTS AND THE HUBBLE CONSTANT
}

\author{
E. M. L. Humphreys ${ }^{1,2}$, M. J. Reid ${ }^{2}$, J. M. Moran ${ }^{2}$, L. J. Greenhill ${ }^{2}$, And A. L. Argon ${ }^{2}$ \\ ${ }^{1}$ European Southern Observatory, Karl-Schwarzschild-Str. 2, D-85748 Garching bei München, Germany; ehumphre @eso.org \\ ${ }^{2}$ Harvard-Smithsonian Center for Astrophysics, 60 Garden Street, Cambridge, MA 02138, USA \\ Received 2012 December 4; accepted 2013 July 22; published 2013 August 29
}

\begin{abstract}
We report a new geometric maser distance estimate to the active galaxy NGC 4258. The data for the new model are maser line-of-sight (LOS) velocities and sky positions from 18 epochs of very long baseline interferometry observations, and LOS accelerations measured from a $10 \mathrm{yr}$ monitoring program of the $22 \mathrm{GHz}$ maser emission of NGC 4258. The new model includes both disk warping and confocal elliptical maser orbits with differential precession. The distance to NGC 4258 is $7.60 \pm 0.17 \pm 0.15 \mathrm{Mpc}$, a $3 \%$ uncertainty including formal fitting and systematic terms. The resulting Hubble constant, based on the use of the Cepheid variables in NGC 4258 to recalibrate the Cepheid distance scale, is $H_{0}=72.0 \pm 3.0 \mathrm{~km} \mathrm{~s}^{-1} \mathrm{Mpc}^{-1}$.
\end{abstract}

Key words: distance scale - galaxies: individual (NGC 4258) - galaxies: nuclei - masers - techniques: interferometric

Online-only material: color figures

\section{INTRODUCTION}

Observations and modeling of masers in the circumnuclear disk of the Seyfert 2/LINER galaxy NGC 4258 have resulted in a distance estimate of $7.2 \pm 0.2$ (random) \pm 0.5 (systematic) Mpc (Herrnstein et al. 1999, hereafter H99), in which the systematic component largely allowed for the potential effects of unmodeled eccentric orbits. The goal of the current work is to reduce this uncertainty (Argon et al. 2007, hereafter Paper I; Humphreys et al. 2005, 2008, hereafter Paper II; Moran et al. 2007). In this paper, we report a new distance estimate for NGC 4258 in which considerably more data have been used: 18 epochs of Very Long Baseline Interferometry (VLBA) data compared with the 4 epochs used in H99. Also, significant progress has been made in the modeling approach, including the possibility of eccentricity in the maser orbits.

NGC 4258 cannot be used to determine the Hubble constant $H_{0}$ directly to high accuracy, since the galaxy is relatively close and its peculiar motion could be a large fraction of its redshift. However, it can be used as an anchor for the Cepheidcalibrated extragalactic distance scale, in addition to the Large Magellanic Cloud and the Milky Way, to reduce uncertainty in $H_{0}$. The Hubble Space Telescope Key Project measured $H_{0}=72 \pm 3 \pm 7 \mathrm{~km} \mathrm{~s}^{-1} \mathrm{Mpc}^{-1}$ (Freedman et al. 2001). Using the current maser distance to NGC 4258 of H99, Macri et al. (2006) recalibrated the Cepheid period-luminosity relation to obtain $H_{0}=74 \pm 3 \pm 6 \mathrm{~km} \mathrm{~s}^{-1} \mathrm{Mpc}^{-1}$. Riess et al. (2011, 2012) also attempted to recalibrate the Cepheid relation using an unpublished preliminary maser distance to NGC 4258 of $7.28 \mathrm{Mpc} \pm 3 \%$, obtaining $H_{0,4258}=74.8 \pm 3.1 \mathrm{~km} \mathrm{~s}^{-1} \mathrm{Mpc}^{-1}$, a fractional accuracy of $4 \%$, where $H_{0,4258}$ is the Hubble constant determined when the sole anchor galaxy is NGC 4258.

We describe the input data for the distance models in Section 2, the models themselves in Section 3, and we compare with the approach of previous work in Section 4. We present the results in Section 5, discuss the impact of the new maser distance on $H_{0}$ in Section 6, and summarize conclusions in Section 7.

\section{INPUT DATA}

The data for our maser geometric distance measurement come from VLBI mapping to obtain maser sky positions, augmented by single dish monitoring of spectra to measure centripetal accelerations. The data used to determine the maser disk geometry and the distance to NGC 4258 consist of maser emission positions $(X, Y)$, line-of-sight (LOS) velocities $\left(v_{\text {los }}\right)$, and LOS accelerations $\left(a_{\text {los }}\right)$. We measured position and velocity data at 18 epochs using VLBI with the methods described in Paper I. We also estimated accelerations (from time-varying Doppler shifts) from spectra obtained during the VLBI observations, supplemented by spectra from the Jansky Very Large Array $\left(\mathrm{VLA}^{3}\right)$ and the Robert C. Byrd Green Bank Telescope (GBT). The acceleration estimates were documented in Paper II. The resulting data set consisted of $\sim 10,000$ data points. In order to create a more tractable data set to use in the disk-fitting programs described here, we binned the data acquired at different epochs in velocity (using a bin width of $1 \mathrm{~km} \mathrm{~s}^{-1}$ ) yielding the reduced data set described in Table 1.

The entries in Table 1 are listed separately for the high-velocity redshifted and blueshifted maser emission occurring at about $v_{\text {sys }} \pm 1000 \mathrm{~km} \mathrm{~s}^{-1}$, respectively, where $v_{\text {sys }}$ is the galactic systemic velocity, and for the low-velocity (systemic) maser emission occurring at about $v_{\text {sys }}$. We give the range of LOS velocities measured for maser emission over all epochs of the observations, and the associated ranges of maser $X$ and $Y$ sky positions from VLBI observations. The LOS accelerations for the maser emission were determined using both single-dish and interferometric data using a Gaussian decomposition method that simultaneously fit Gaussians to maser spectra at multiple epochs to determine drifts in velocity over time. The number of data points in the reduced data set is also provided separately for the high-velocity and low-velocity emission in Table 1.

\footnotetext{
3 The National Radio Astronomy Observatory is a facility of the National Science Foundation operated under cooperative agreement by Associated Universities, Inc.
} 
Table 1

Input Data Set

\begin{tabular}{|c|c|c|c|}
\hline & Reds & Systemics & Blues \\
\hline Number of data points ${ }^{\mathrm{a}}$ & 151 & 187 & 32 \\
\hline$v_{\text {los }}$ range $\left(\mathrm{km} \mathrm{s}^{-1}\right)^{\mathrm{b}}$ & {$[1227.5,1647.5]$} & {$[382.5,577.5]$} & {$[-516.5,-280.5]$} \\
\hline $\boldsymbol{X}$ range $(\mathrm{mas})^{\mathrm{c}}$ & {$[7.774,2.776]$} & {$[-0.513,0.245]$} & {$[-4.51,-8.297]$} \\
\hline $\boldsymbol{Y}$ range $(\text { mas })^{\mathrm{c}}$ & {$[0.134,1.195]$} & {$[-0.112,0.092]$} & {$[-0.014,1.060]$} \\
\hline$a_{\text {los }}$ range $\left(\mathrm{km} \mathrm{s}^{-1} \mathrm{yr}^{-1}\right)$ & {$[-0.40,0.73]$} & {$[6.96,9.81]$} & {$[-0.72,0.04]$} \\
\hline
\end{tabular}

Notes.

${ }^{\text {a }}$ Number of data points refers to $X, Y$, and $v_{\operatorname{los}}$ data. $a_{\text {los }}$ data were not measured for each of these points.

b Velocities are radio definition and LSR reference frame.

${ }^{c}$ Positions are measured relative to that of maser emission at $510 \mathrm{~km} \mathrm{~s}^{-1}$.

\section{3D DISK-FITTING MODEL}

\subsection{Overview of the Model}

We employed a Bayesian three-dimensional (3D) global diskfitting program (Reid et al. 2013) in order to determine the maser disk geometry and estimate distance (Section 3.2). Our model has 13 global parameters, which we describe in the following paragraphs.

The global disk parameters include distance $(D)$, black hole mass $\left(M_{\mathrm{bh}}\right)$, galaxy systemic velocity $\left(v_{0}\right)$, black hole $x$-position $\left(X_{0}\right)$, and $y$-position $\left(Y_{0}\right)$.

A simple model for elliptical maser orbits is one in which eccentric orbits precess coherently in the disk, such that the orbits are aligned and nested (Statler 2001, 2002). This scenario could be described by a single eccentricity $(e)$ and a periapsis angle $(\omega)$. However, it may not be a valid description for the nuclear maser disk, since zero viscosity would be required and there is no mechanism by which such a disk would accrete. Therefore, the model we investigated is one for a viscous disk in which orbits undergo differential precession and the periapsis angle is described by a leading/trailing spiral (Armitage 2008). This introduces a radial gradient in the periapsis angle $\omega$, such that $\omega_{r}=\omega_{0}+r d \omega / d r$, where the reference angular radius for $\omega_{0}$ is $r=0$.

We modeled the warped disk as a surface whose position angles and inclinations vary as smooth functions of radius $\left(\Omega_{r}, i_{r}\right)$. Herrnstein et al. (2005) found that the warp is well described by an inclination warp of $i_{r}=i_{0}+r d i / d r$ and a position angle warp of $\Omega_{r}=\Omega_{0}+r d \Omega / d r+r^{2} d^{2} \Omega / d r^{2}$, where in this paper the reference radius for $i_{0}$ and $\Omega_{0}$ is $r=0$. However, we investigated the effect on the distance of inclusion of a second-order inclination warp term as part of our quantification of systematic uncertainties in Section 5.2. The disk inclination angle is measured between the observer's LOS and the negative spin axis of the maser disk; the position angle is measured east of north.

In addition to the 13 global disk parameters, the angular radius $(r)$ and the azimuth angle $(\phi)$ in the disk of each maser spot were included as parameters. In total, there were 753 parameters in the model, which we fitted to 1262 data points.

The geometry of the sky and disk coordinate systems, and the derivation of the equations used in the modeling, are described in Appendix A. In the modeling, we assumed that masers orbit a point mass located at the focus of confocal ellipses of common eccentricity.
The model LOS velocities for masers (Appendix B) are given by Keplerian rotation, for which

$$
v_{\text {los, model }}=v_{r} \sin i_{r} \sin \phi+v_{\gamma} \sin i_{r} \cos \phi+v_{0},
$$

where $v_{r}$ and $v_{\gamma}$ are the radial and tangential components given by $v_{r}=\left[G M_{\mathrm{bh}} /(r D(1+e \cos \gamma))\right]^{1 / 2} e \sin \gamma$ and $v_{\gamma}=$ $\left[G M_{\mathrm{bh}}(1+e \cos \gamma) /(r D)\right]^{1 / 2}$, where $\gamma=\phi-\omega_{r}$ is the angle between the maser and perihelion. However, due to significant special and general relativistic effects in the transformation of observed frequency to the LOS velocity for the masers, we used relativity-corrected LOS velocities $v_{\text {los,model }}^{\prime}$ (Appendix C).

The component of centripetal acceleration in the LOS is given by

$$
a_{\mathrm{los}, \text { model }}=\frac{-G M_{\mathrm{bh}}}{(r D)^{2}} \sin i_{r} \sin \phi,
$$

and the sky positions of masers are given by

$$
\begin{aligned}
& X=r\left(\sin \Omega_{r} \cos \phi-\cos \Omega_{r} \cos i \sin \phi\right)+X_{0} \\
& Y=r\left(\cos \Omega_{r} \cos \phi+\sin \Omega_{r} \cos i \sin \phi\right)+Y_{0},
\end{aligned}
$$

where $\left(X_{0}, Y_{0}\right)$ is the sky position of the disk dynamical center measured relative to maser emission at $510 \mathrm{~km} \mathrm{~s}^{-1}$. It is important to note that this reference position in the maser disk is defined by velocity, not by a specific clump sometimes called a maser "spot" or "feature."

In the modeling, we compared observed maser ( $X_{\mathrm{obs}}, Y_{\mathrm{obs}}$, $\left.v_{\mathrm{obs}}^{\prime}, a_{\mathrm{obs}}\right)$ with model values ( $\left.X_{\text {model }}, Y_{\text {model }}, v_{\mathrm{los}, \text { model }}^{\prime}, a_{\mathrm{los}, \text { model }}\right)$ to determine distance. Essentially, the rotation curve of the high-velocity maser emission constrains $\mathcal{M}^{1 / 2} \sin i_{r}$ where $\mathcal{M}=\left(M_{\mathrm{bh}} / D\right)$ and the accelerations of the systemic maser features provide distance via $D=\left(-G \mathcal{M} / r^{2} a_{\text {los,model }}\right) \sin i_{r} \sin \phi$.

\subsection{The Bayesian Fitting Program}

We used a Markov Chain Monte Carlo (MCMC) fitting program in which the Metropolis-Hastings algorithm was used to select the Markov-chain trial parameters. We used an initial 2,000,000 "burn-in" trials to be discarded at the start of each fitting run and then saved the outcome of the subsequent 10,000,000 trials. The MCMC parameter step size was adjusted every 100,000 steps during burn-in in order to maintain an acceptance rate of $25 \%$ of the trial parameters. The trial values for each parameter were binned, yielding marginalized posteriori probability distributions, from which we quote median values and $68 \%$ confidence (" $1 \sigma$ ") ranges.

The MCMC method is not designed to yield the "best" minimum in $\chi^{2}$ space. However, we output the lowest $\chi^{2}$ of the MCMC trials, calculated using

$$
\begin{aligned}
\chi^{2}= & \sum_{n}\left(\frac{v_{\text {obs }}^{\prime}-v_{\text {los, model }}^{\prime}}{\sigma_{v}}\right)^{2}+\left(\frac{X_{\text {obs }}-X_{\text {model }}}{\sigma_{x}}\right)^{2} \\
& +\left(\frac{Y_{\text {obs }}-Y_{\text {model }}}{\sigma_{y}}\right)^{2}+\left(\frac{a_{\text {obs }}-a_{\text {los, } \text { model }}}{\sigma_{a}}\right)^{2}
\end{aligned}
$$

and scaled the $1 \sigma$ uncertainties for each global disk parameter by $\sqrt{\chi^{2} / N}$, where $N$ is the number of degrees of freedom.

\section{COMPARISON WITH PREVIOUS APPROACHES}

There are several notable differences between the current work and that of H99. First, in H99, the distance calculation was 
Table 2

High-velocity Maser Feature Fit of Herrnstein et al. (2005)

\begin{tabular}{lc}
\hline \hline Parameter & Model Value \\
\hline Distance, $D(\mathrm{Mpc})$ & {$[7.2]^{\mathrm{a}}$} \\
Black hole mass, $M_{\mathrm{bh}}\left(\times 10^{7} M_{\odot}\right)$ & 3.79 \\
Galaxy systemic velocity, $v_{\text {sys }}\left(\mathrm{km} \mathrm{s}^{-1}\right)$ & 473.5 \\
Dynamical center $x$-position, $X_{0}^{\mathrm{b}}(\mathrm{mas})$ & -0.19 \\
Dynamical center $y$-position, $Y_{0}{ }^{\mathrm{b}}(\mathrm{mas})$ & {$[0.55]^{\mathrm{a}}$} \\
Inclination, $i_{0}(\mathrm{deg})$ & {$[73.80]^{\mathrm{a}}$} \\
Inclination warp, $d i / d r\left(\mathrm{deg}\right.$ mas $\left.^{-1}\right)$ & 1.95 \\
Position angle, $\Omega_{0}(\mathrm{deg})$ & 65.65 \\
Position angle warp, $d \Omega / d r\left(\mathrm{deg} \mathrm{mas}^{-1}\right)$ & 5.04 \\
Position angle warp, $d^{2} \Omega / 2 d r^{2}\left(\mathrm{deg}^{\mathrm{m}} \mathrm{mas}^{-2}\right)$ & -0.13 \\
\hline
\end{tabular}

Notes.

a Values were adopted, not fitted.

${ }^{\mathrm{b}}$ Positions are measured relative to that of maser emission at $510 \mathrm{~km} \mathrm{~s}^{-1}$.

performed in two steps. A warped disk model was determined without the incorporation of systemic feature accelerations. Distance was then calculated in a decoupled second step involving these accelerations. In the current work, we fitted the data in a single step, simultaneously adjusting all parameters. The second difference is that, in $\mathrm{H} 99, r$ and $\phi$ were not treated as model parameters with associated uncertainties. In their model, allowed loci of $(r, \phi)$ were determined from LOS velocities, and $r$ and $\phi$ were assumed to be perfectly determined. In our work, maser emission $r$ and $\phi$ are included as parameters in the models. The third difference is that orbits were fixed to be circular in H99, whereas in the current model, eccentric maser orbits are included.

Another difference between this work and the investigation of disk warping performed by Herrnstein et al. (2005) is that they only performed a fit to the high-velocity maser emission. In this work, we fit to all observables for both the low-velocity (systemic) and the high-velocity emission.

Finally, the distance estimate of H99 was based on 4 VLBI epochs, whereas we analyze $18 \mathrm{VLBI}$ epochs. The acceleration data set of $\mathrm{H} 99$ included accelerations for fewer than 20 systemic maser features measured from 4 epochs over $3 \mathrm{yr}$. In the current work, we included acceleration measurements for both systemic and high-velocity features from a monitoring program lasting up to $\sim 10 \mathrm{yr}$, described in detail in Paper II of this series.

\section{MASER DISTANCE TO NGC 4258}

\subsection{The "Base" Model}

We used the MCMC program to establish an optimum or "base" model for the NGC 4258 maser disk geometry and distance. The initial global disk parameters to the fitting program were taken to be approximately those given by Herrnstein et al. (2005, Table 2). In order to ensure that the eccentricity space was fully explored, we set initial values of eccentricity as high as 0.5. All global disk parameters had flat priors. Input values for the $(r, \phi)$ maser feature polar disk coordinate parameters were estimated using observational data and assuming Keplerian rotation. These parameters were assigned the following loose prior uncertainties: in the range $\pm \sim 1-2$ mas and $\pm 20^{\circ}$ for the high-velocity $(r, \phi)$. Systemic features had prior uncertainties of \pm 2 mas and $\pm 1^{\circ}$.

Inspection of data among different observing epochs showed scatter larger than expected from the formal uncertainties. In order to account for this, error floors were added to the formal
Table 3

The Distance Base Model

\begin{tabular}{lc}
\hline \hline Parameter & Value $^{\mathrm{a}}$ \\
\hline Distance, $D(\mathrm{Mpc})$ & $7.60 \pm 0.17$ \\
Black hole mass, $M_{\mathrm{bh}}\left(\times 10^{7} M_{\odot}\right)$ & $4.00 \pm 0.09$ \\
Galaxy systemic velocity, $v_{\text {sys }}\left(\mathrm{km} \mathrm{s}^{-1}\right)$ & $474.25 \pm 0.49$ \\
Dynamical center $x$-position, $X_{0}^{\mathrm{b}}(\mathrm{mas})$ & $-0.204 \pm 0.005$ \\
Dynamical center $y$-position, $Y_{0}^{\mathrm{b}}(\mathrm{mas})$ & $0.560 \pm 0.006$ \\
Inclination, $i_{0}(\mathrm{deg})$ & $71.74 \pm 0.48$ \\
Inclination warp, $d i / d r\left(\mathrm{deg}\right.$ mas $\left.^{-1}\right)$ & $2.49 \pm 0.11$ \\
Position angle, $\Omega_{0}(\mathrm{deg})$ & $65.46 \pm 0.98$ \\
Position angle warp, $d \Omega / d r\left(\mathrm{deg}^{-1} \mathrm{mas}^{-1}\right)$ & $5.23 \pm 0.30$ \\
Position angle warp, $d^{2} \Omega / 2 d r^{2}\left(\mathrm{deg} \mathrm{mas}^{-2}\right)$ & $-0.24 \pm 0.02$ \\
Eccentricity, $e$ & $0.006 \pm 0.001$ \\
Periapsis angle, $\omega_{0}(\mathrm{deg})$ & $293.5 \pm 64.4$ \\
Periapsis angle warp, $d \omega / d r\left(\mathrm{deg}^{-2}\right.$ mas $\left.^{-1}\right)$ & $59.5 \pm 10.2$ \\
\hline
\end{tabular}

Notes.

${ }^{\mathrm{a}}$ Uncertainties have been scaled by $\sqrt{\chi^{2} / N}$ i.e., $\sqrt{1.403}$.

b Positions are measured relative to that of maser emission at $510 \mathrm{~km} \mathrm{~s}^{-1}$.

uncertainties in quadrature. The error floors effectively weight data differently in the MCMC modeling, which is somewhat subjective, but which is investigated as part of our analysis of systematic uncertainties in the modeling. The error floors used here were 0.02 and 0.03 mas for maser $x$ and $y$ sky positions, respectively (VLBI position uncertainties are larger in the $y$ data due to north-south beam elongation of a factor of 1.5 for NGC 4258 at a declination of $+47^{\circ}$ ), $1.0 \mathrm{~km} \mathrm{~s}^{-1}$ for all maser feature velocity error floors, and $0.3 \mathrm{~km} \mathrm{~s}^{-1} \mathrm{yr}^{-1}$ for acceleration data. The impact of selection of error floors on distance is investigated in Section 5.2 as part of our quantification of systematic uncertainties. We note that it is important that the high-velocity maser features have sufficient weight so as to constrain the rotation curve, since it provides the strongest constraints on $M_{\mathrm{bh}} / D$.

The resulting base model (Table 3 and Figures 1-7) has parameters for the maser disk warping that are in good agreement with those found in the fit to high-velocity maser features only by Herrnstein et al. (2005). The eccentricity of maser orbits is low at $0.006 \pm 0.001$. We estimate a maser distance of $7.60 \pm 0.17 \mathrm{Mpc}$ (formal fitting error scaled by $\sqrt{\chi^{2} / N}$ where $\left.\chi^{2} / N=1.4\right)$.

\subsection{Investigation of Systematic Uncertainties}

In addition to the formal uncertainty derived directly from the MCMC fitting program, we investigated various sources of systematic uncertainty that could affect the maser distance to NGC 4258. First, we varied the values of the error floors that we added in quadrature to the formal errors on our data set to assess their impact on distance. Second, we investigated the effect of changing the initial parameter values and the random number generator seed used for selecting MCMC trial disk parameters. In particular, we ran the MCMC trials for a wide range of input distances $(7.1-8.2 \mathrm{Mpc})$ to make sure that no memory of the starting value was retained. Third, we tested the impact on distance if we allowed a second-order inclination warp term and what would happen if we constrained the maser orbits to be circular. The results of these tests are presented in Table 4.

In Table 4, we found that the largest sources of systematic uncertainty in the distance estimation are given by starting the MCMC code with different initial values for distance 

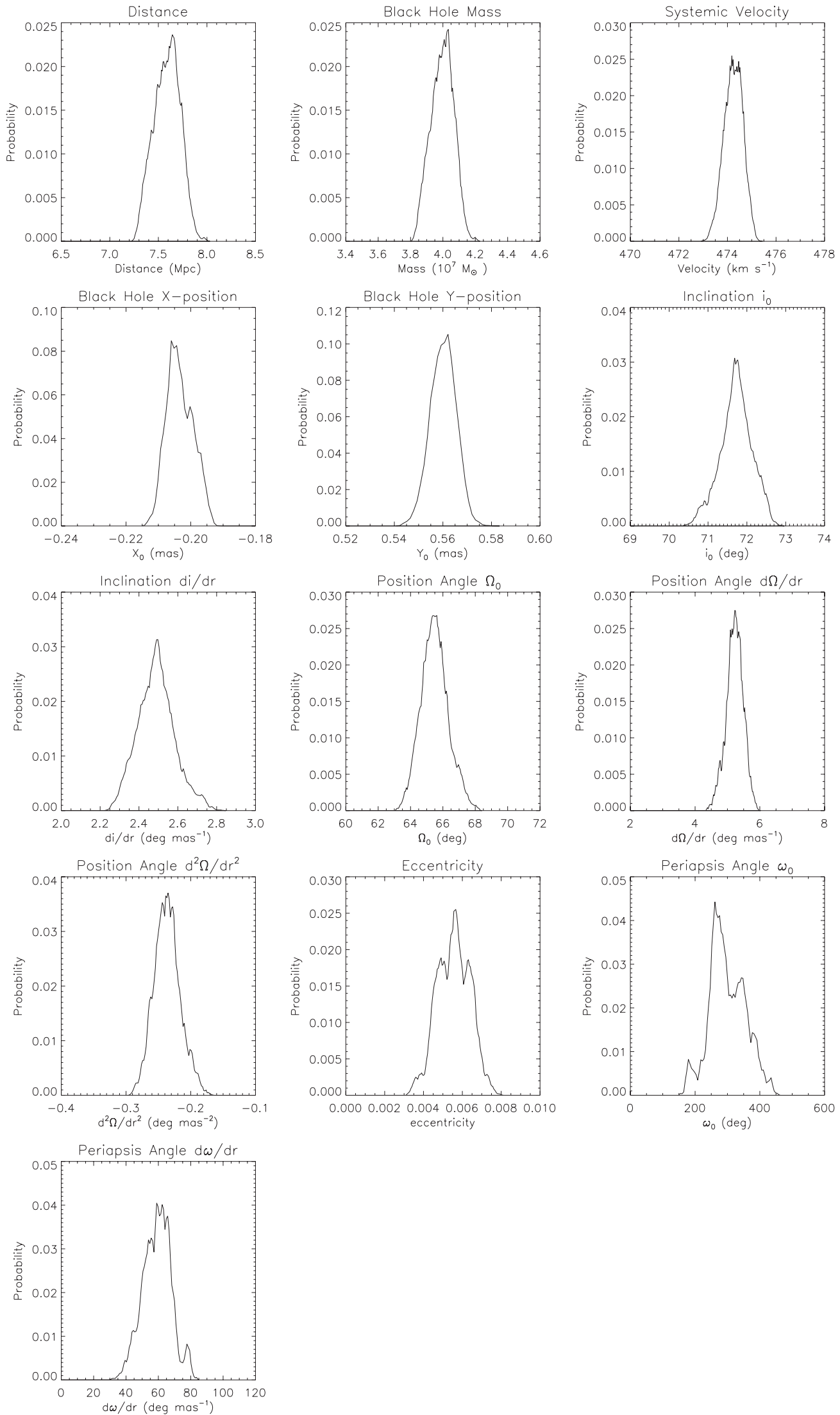

Figure 1. Marginalized posteriori probability distributions for the global disk parameters describing the distance base model. These have been produced by binning 10,000,000 MCMC trial values for each parameter. 
Table 4

Table of Distance Uncertainty Terms

\begin{tabular}{|c|c|c|c|c|}
\hline \multirow[t]{2}{*}{ Formal uncertainty } & & \multirow{2}{*}{$\begin{array}{l}\text { Distance } \\
(\mathrm{Mpc})\end{array}$} & \multicolumn{2}{|c|}{$1 \sigma$ Uncertainty } \\
\hline & & & $(\mathrm{Mpc})$ & $(\%)$ \\
\hline Base model & & 7.596 & \pm 0.167 & 2.20 \\
\hline \multirow[t]{2}{*}{ Systematic uncertainties } & \multirow[t]{2}{*}{$\begin{array}{c}\text { Value in } \\
\text { Base Model }\end{array}$} & Distance & \multicolumn{2}{|c|}{$\begin{array}{c}\Delta D \text { from Base Model } \\
\text { or rms Estimate }\end{array}$} \\
\hline & & $(\mathrm{Mpc})$ & $(\mathrm{Mpc})$ & $(\%)$ \\
\hline Different $h v$-feature velocity uncertainties $\left(0.7\right.$ and $\left.1.3 \mathrm{~km} \mathrm{~s}^{-1}\right)$ & $1.0 \mathrm{~km} \mathrm{~s}^{-1}$ & 7.624 & 0.028 & 0.37 \\
\hline Different $y$-error floor $(20 \mu$ as $)$ & $30 \mu$ as & 7.639 & 0.043 & 0.57 \\
\hline Different acceleration error floor $\left(0.5 \mathrm{~km} \mathrm{~s}^{-1} \mathrm{yr}^{-1}\right)$ & $0.3 \mathrm{~km} \mathrm{~s}^{-1} \mathrm{yr}^{-1}$ & 7.564 & -0.032 & 0.42 \\
\hline Different initial conditions $^{\mathrm{a}}$ & $\ldots$ & $\ldots$ & \pm 0.114 & 1.50 \\
\hline Assuming eccentricity is zero & $\ldots$ & 7.619 & 0.023 & 0.30 \\
\hline Inclusion of $d^{2} i / d r^{2}$ term (solves to $-0.112 \pm 0.034 \mathrm{deg} \mathrm{mas}^{-2}$ ) & $\ldots$ & 7.562 & -0.034 & 0.45 \\
\hline Unmodeled spiral structure & $\cdots$ & $\cdots$ & \pm 0.076 & 1.00 \\
\hline Systematic uncertainties added in quadrature & & & 0.15 & $2.0 \%$ \\
\hline Uncertainty in the maser distance to NGC $4258^{\text {b }}$ & & & 0.23 & $3.0 \%$ \\
\hline
\end{tabular}

Notes.

${ }^{a}$ This includes different seeds in the random number generator and different initial distances in the model.

${ }^{b}$ The final uncertainty is calculated by adding individual sources of uncertainty in quadrature.
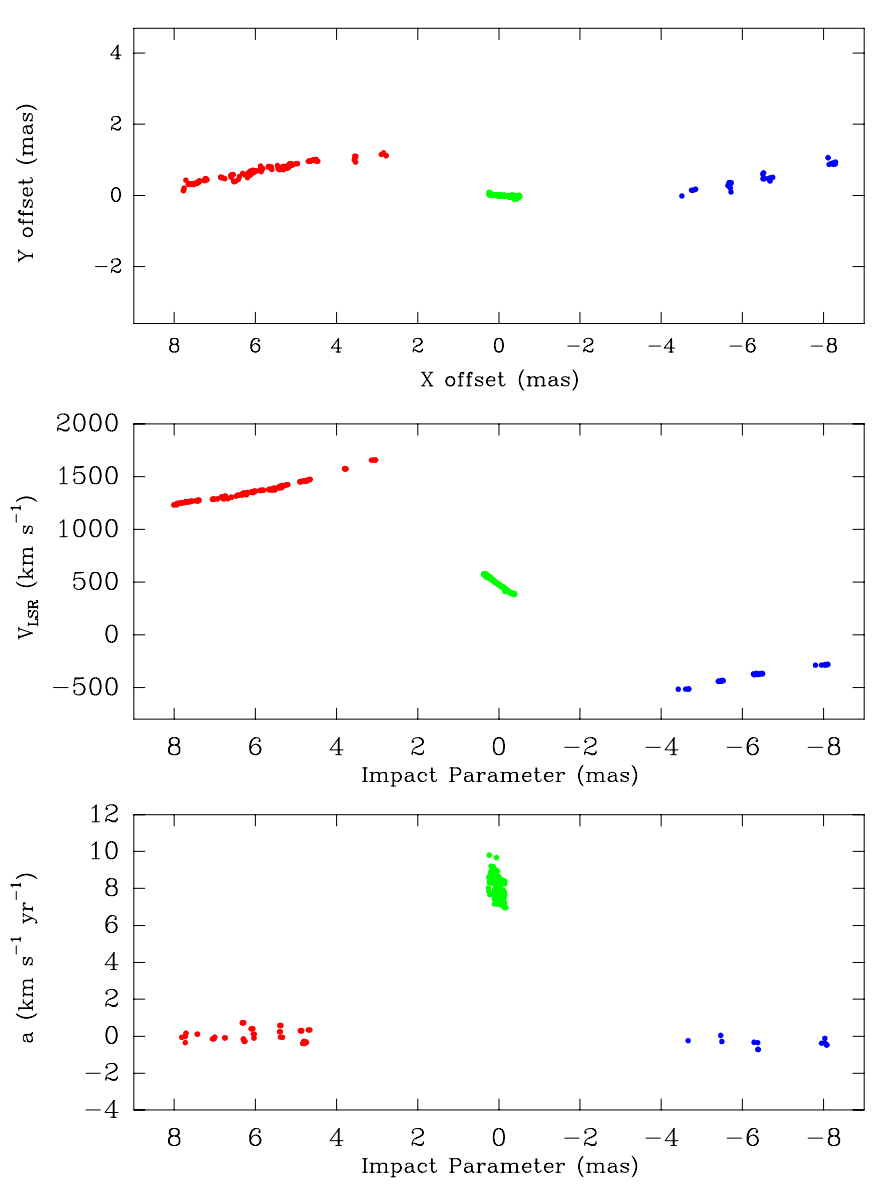

Figure 2. Input data to the model: maser sky positions (top), $P-V$ diagram (middle), and accelerations (bottom).

(A color version of this figure is available in the online journal.)

which contributes $1.5 \%$, and the possibility of unmodeled spiral structure (Section 5.2.1) which contributes $\sim 1 \%$. Other sources of systematic uncertainty were all found to cause $<1 \%$ change in distance. Summing the uncertainty terms in quadrature gives a total systematic uncertainty of $\pm 0.15 \mathrm{Mpc}$ or $\pm 2 \%$.
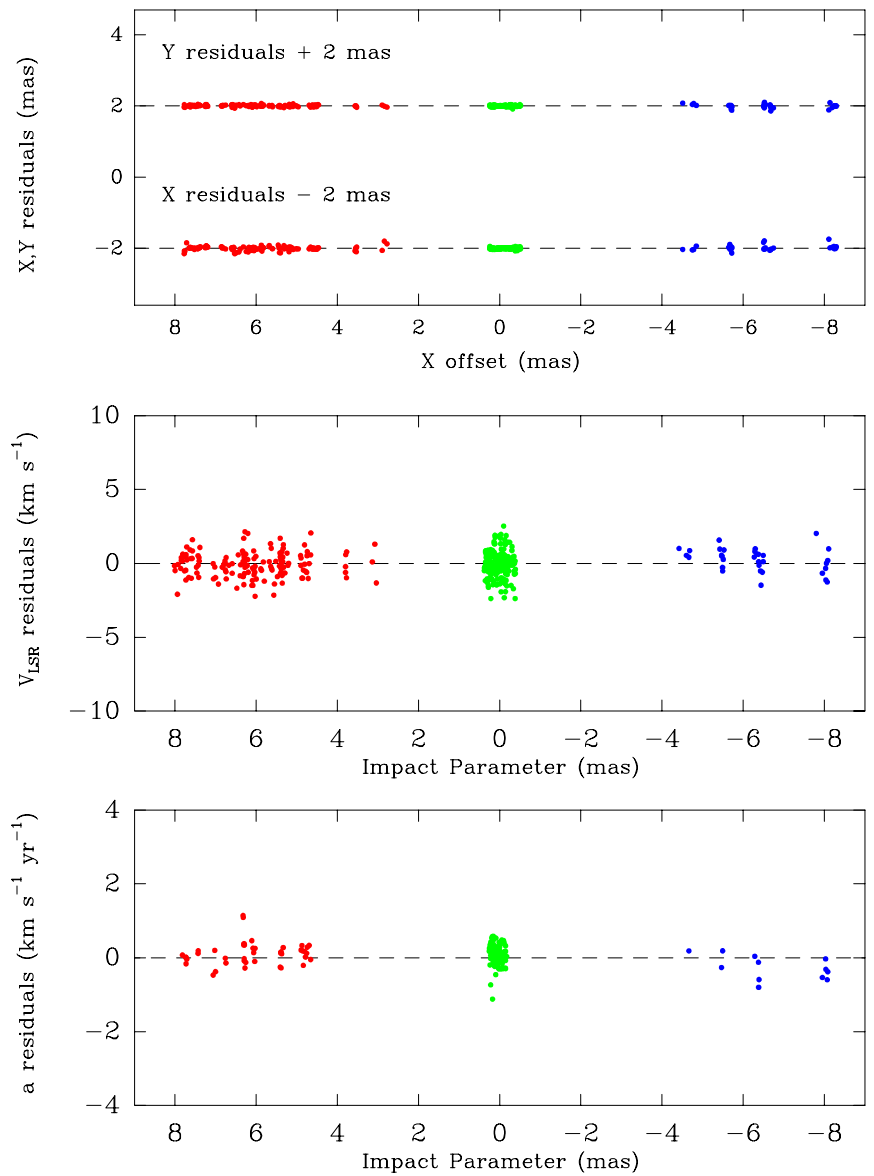

Figure 3. Residuals from the base model fit to the data: maser sky position residuals (top), offset by \pm 2 mas for clarity; velocity residuals (middle); and acceleration residuals (bottom).

(A color version of this figure is available in the online journal.)

\subsubsection{Systematic Uncertainty Due to Unmodeled Spiral Structure}

In Paper II, we showed evidence for a periodic structure in the NGC 4258 high-velocity maser emission feature position distribution (originally noted by Maoz 1995). We also found a 


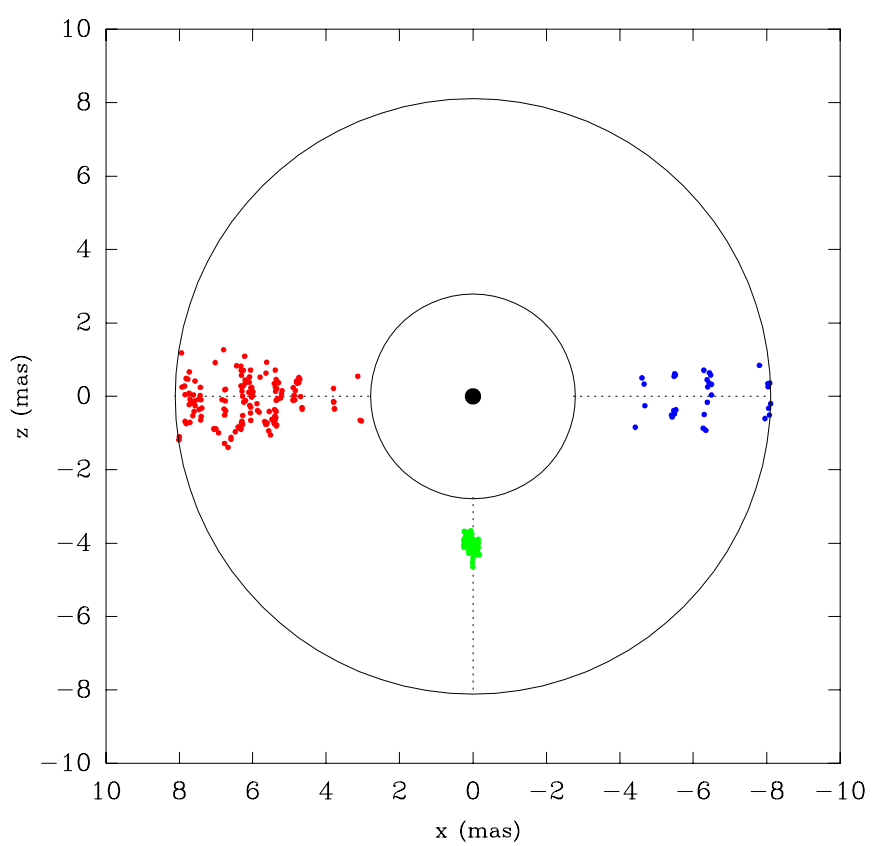

Figure 4. View of the disk plane for the base model. The deprojected maser positions are shown in red, green, and blue for the redshifted, systemic, and blueshifted masers, respectively.

(A color version of this figure is available in the online journal.)

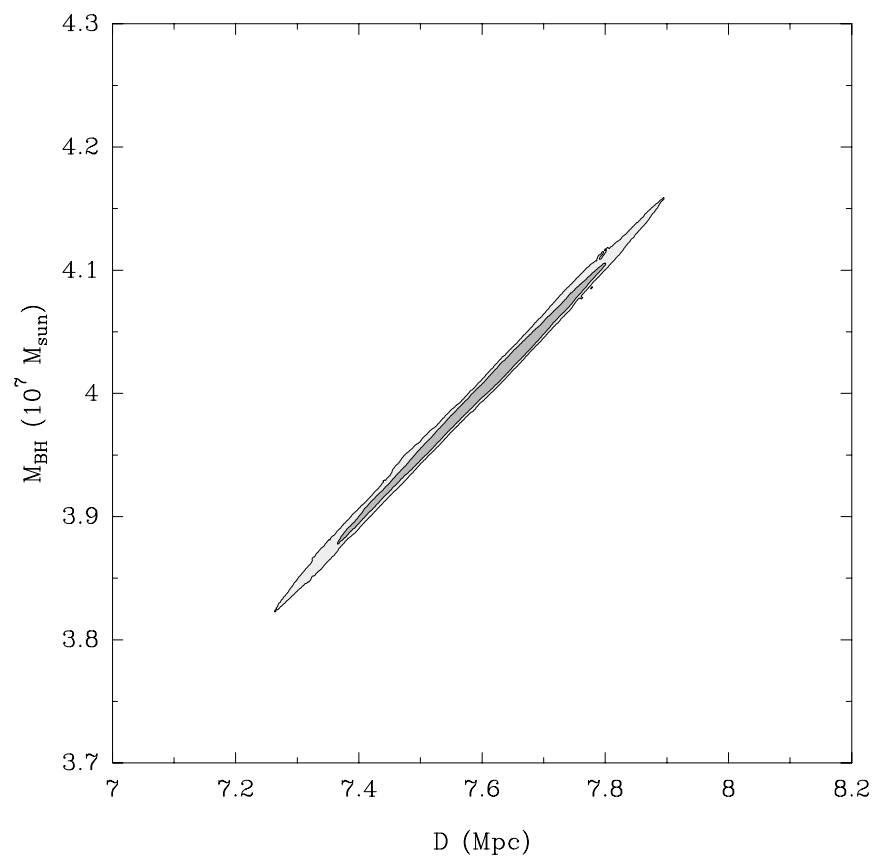

Figure 5. 2D histogram of distance and mass outcomes from the $10,000,000$ MCMC trials. The bin sizes used to compute the plot were $0.001 \mathrm{Mpc}$ and $0.001 M_{\odot}$, respectively. Grayscale contours code the $68 \%$ and $95 \%$ confidence intervals.

persistent slope in the LOS accelerations of low-velocity maser emission as a function of the disk impact parameter. The slope was first seen by Greenhill et al. (1995) and so has persisted for at least 6-7 yr. This is notable because if it were just an "accidental" quirk in the radial distribution of systemic maser features, it should have systematically moved or rotated out of the LOS within a few years. However, both of these results could be explained by the presence of spiral structure in the disk.

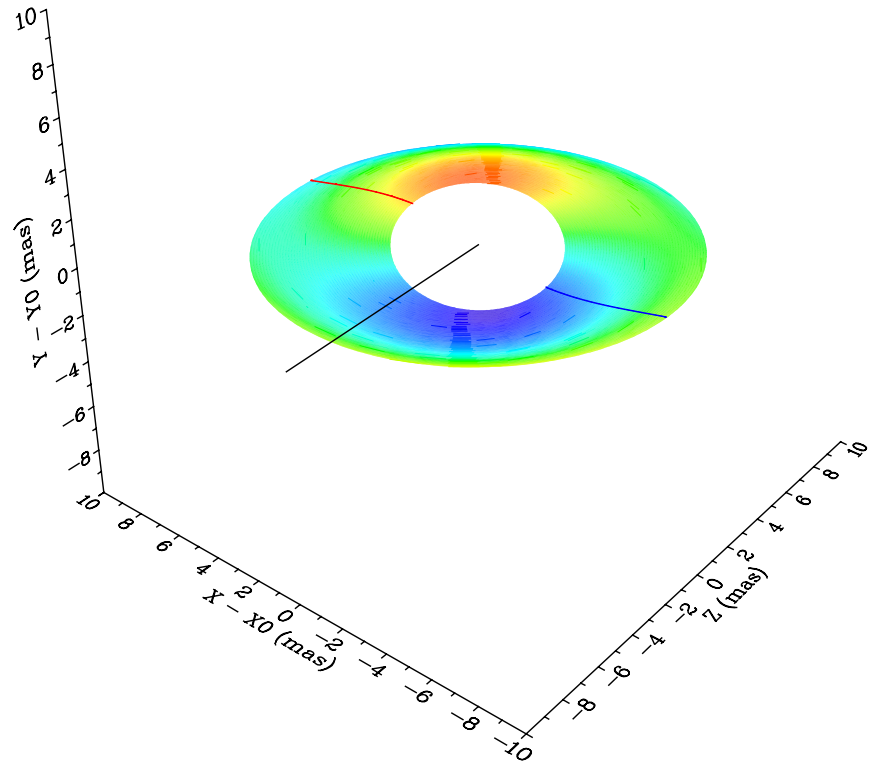

Figure 6. Base model maser disk seen from a viewpoint at $[-40,40,-50]$ mas The LOS is shown as a line extending beyond the outer edge of the disk in black along the $Z$-direction. The solid color contours show disk elevation, where red is the maximum and dark blue is the minimum. The disk midline is shown for redshifted emission (red line) and blueshifted emission (blue line), respectively. The low-velocity (systemic) masers lie in the concavity on the front side of the disk.

(A color version of this figure is available in the online journal.)
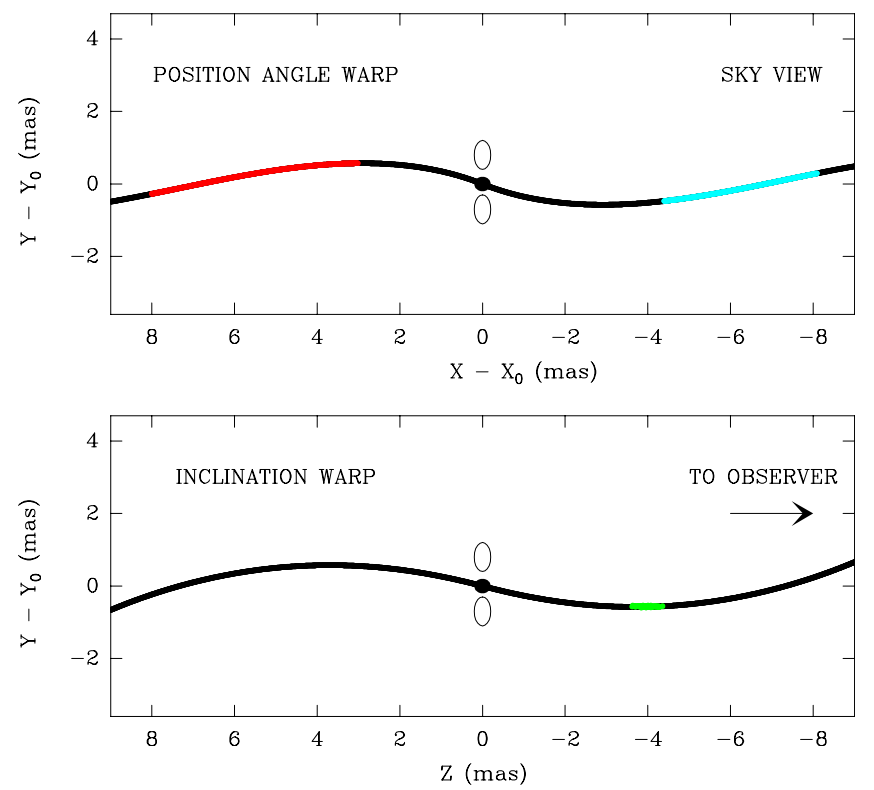

Figure 7. Cross-sections of the warped disk of the base model. Top: cut of the disk along the disk midline, showing the position angle warp given by $\Omega_{r}=\Omega_{0}+r d \Omega / d r+r^{2} d^{2} \Omega / d r^{2}$. The total extents of red- and blueshifted maser regions from the base model are overplotted. Bottom: cut of the disk along the LOS to the black hole, showing the inclination warp given by $i_{r}=i_{0}+r d i / d r$. The total extent of the systemic maser region (for systemic masers with acceleration measurements) from the base model is overplotted. Both panels: the northern oval represents the observed position of northern radio jet emission (Herrnstein et al. 1997) and the southern oval is a reflection of the northern one through the disk center (following Herrnstein et al. 2005).

(A color version of this figure is available in the online journal.)

Stability arguments support self-gravitating structure formation, at least in the outer disk regions. The Toomre $Q$-parameter calculated for $n\left(\mathrm{H}_{2}\right)=10^{10} \mathrm{~cm}^{-3}$, a full disk height of $12 \mu \mathrm{as}$ (Paper I), and a central black hole mass of $4.0 \times 10^{7} M_{\odot}$ varies 
Table 5

Unmodeled Spiral Structure Test

\begin{tabular}{lcr}
\hline \hline Parameter & Model Value & Fitted Value \\
\hline Distance, $D(\mathrm{Mpc})$ & 7.20 & 7.46 \\
Black hole mass, $M_{\mathrm{bh}}\left(\times 10^{7} M_{\odot}\right)$ & 3.80 & 3.96 \\
Galaxy systemic velocity, $v_{\text {sys }}\left(\mathrm{km} \mathrm{s}^{-1}\right)$ & 474.00 & 436.20 \\
Dynamical center $x$-position, $X_{0}(\mathrm{mas})$ & 0.000 & 0.17 \\
Dynamical center $y$-position, $Y_{0}(\mathrm{mas})$ & 0.000 & 0.00 \\
Inclination, $i_{0}(\mathrm{deg})$ & 90.0 & 89.97 \\
Position angle, $\Omega_{0}(\mathrm{deg})$ & 90.0 & 90.00 \\
Eccentricity, $e$ & 0.00 & 0.078 \\
Periapsis angle, $\omega_{0}(\mathrm{deg})$ & 0.00 & -33.13 \\
Periapsis angle gradient, $d \omega / d r\left(\mathrm{deg} \mathrm{mas}^{-1}\right)$ & 0.00 & 13.57 \\
\hline
\end{tabular}

between 10 and 1 , respectively, for the maser disk between 0.1 and $0.30 \mathrm{pc}$ (however, we note that $Q$ would be between 26 and 3 if the velocity dispersion is used in place of the sound speed). Although $Q<1$ is required for instability to axisymmetric perturbations, instability to non-axisymmetric perturbations occurs in the $1<Q<2$ regime and a spiral structure could form. In Paper II, we performed "proof-of-concept" $N$-body modeling that showed that the gradient in the low-velocity acceleration data could be reproduced by a spiral arm of mass $15 \%$ of the upper limit mass for the maser disk given by Herrnstein et al. (2005).

To assess the impact of unmodeled spiral structure on a distance estimate, we took the same simulated data that reproduced the gradient in acceleration data in Paper II and fed it into our fitting program to see how well it could retrieve the distance. We found that the presence of the spiral arm manifested itself as a significant shift in the recovered black hole $X_{0}$ position by $\sim 0.2$ mas, the galaxy systemic velocity by $\sim 40 \mathrm{~km} \mathrm{~s}^{-1}$, eccentricity by $\sim 0.08$, and the distance by $3.6 \%$ (Table 5 ).

We know that such effects cannot be "hidden" in our data and modeling. In our modeling, we obtain a systemic velocity of $474.3 \pm 0.5 \mathrm{~km} \mathrm{~s}^{-1}$, which is very close to that independently obtained by Cecil et al. (1992) of $472 \pm 4 \mathrm{~km} \mathrm{~s}^{-1}$ for $\mathrm{H} \alpha$ observations using the Hawaii Imaging Fabry-Perot Interferometer. In addition, we find a much lower eccentricity for the NGC 4258 disk at $0.006 \pm 0.001$ compared with the eccentricity of 0.08 found for the simulated data that included spiral structure.

However, it is possible that a smaller effect, at about the $10 \%-20 \%$ level of that obtained for our spiral structure example (i.e., a change in systemic velocity of about $10 \mathrm{~km} \mathrm{~s}^{-1}$ or an eccentricity of about $0.01-0.02$ ), could perhaps be relevant to the NGC 4258 data set. We therefore adopt a conservative $1 \%$ uncertainty in distance due to unmodeled spiral structure and await further study of spiral structure in active galactic nucleus (AGN) nuclear disks to clarify the best way to assess this term in future work.

\subsection{Parameter Correlations}

The global disk parameter correlation matrix for the base model is given in Table 6 . The correlation coefficient of $D$ and $M_{\text {bh }}$ of 0.998 superficially suggests that these parameters are degenerate and calls into question whether these values can be determined reliably from the modeling. However, this naturally occurs when combining constraints on $M_{\mathrm{bh}}$ and $D$ from different types of data that are sensitive to different powers of these parameters. In such a case, while $M_{\mathrm{bh}}$ and $D$ are highly correlated over a range of values, their probable values are also strongly bounded.

In order to investigate how $M_{\mathrm{bh}}$ and $D$ are constrained, consider an edge-on disk with systemic maser features at a fixed angular radius $r_{\text {sys }}$ and high-velocity maser features that lie on the disk midline and at disk angular radii of $r_{\mathrm{hv}}$. We can then formulate three mass-distance relations. The first is from the high-velocity feature Keplerian rotation curve and is $M=G M_{\mathrm{bh}}=W D$, where $W=v_{\mathrm{hv}}^{2} r_{\mathrm{hv}}$. The second is from the slope of the systemic feature position-velocity diagram, with slope $s=M^{0.5} r_{\text {sys }}^{-0.5} D$. The third is that the LOS component of accelerations of the systemic features is given by $a=M r_{\text {sys }}^{-2}$, so that

$$
\begin{aligned}
M & =W D, \\
& =s^{2} r_{\mathrm{sys}}^{3} D^{-2}, \\
& =a r_{\mathrm{sys}}^{2} .
\end{aligned}
$$

Eliminating $r_{\text {sys }}$ from Equations (6) and (7) gives

$$
M=s^{-4} a^{3} D^{4},
$$

obtained only from systemic feature accelerations and the slope of the systemic feature rotation curve. That leaves Equation (5). Plotting Equations (5) and (8) in $M_{\mathrm{bh}}-D$ space yields intersecting curves and, if all the quantities were exactly known, an exact

\begin{tabular}{|c|c|c|c|c|c|c|c|c|c|c|c|c|c|}
\hline Parameter & $D$ & $M_{\mathrm{bh}}$ & $v_{\text {sys }}$ & $x_{0}$ & $y_{0}$ & $i_{0}$ & $d i / d r$ & $\Omega$ & $d \Omega / d r$ & $d^{2} \Omega / 2 d r^{2}$ & $e$ & $\omega$ & $\overline{d \omega / d r}$ \\
\hline $\bar{D}$ & 1.000 & 0.998 & 0.011 & -0.009 & 0.030 & -0.089 & 0.108 & 0.094 & -0.090 & 0.089 & -0.060 & 0.301 & -0.28 \\
\hline$M_{\mathrm{bh}}$ & 0.998 & 1.000 & -0.023 & -0.026 & 0.025 & -0.083 & 0.102 & 0.098 & -0.095 & 0.095 & -0.058 & 0.311 & -0.298 \\
\hline$v_{\text {sys }}$ & 0.011 & -0.023 & 1.000 & 0.062 & 0.210 & -0.124 & 0.119 & -0.216 & 0.240 & -0.239 & 0.051 & -0.413 & 0.42 \\
\hline$Y_{0}$ & 0.030 & 0.025 & 0.210 & -0.115 & 1.000 & -0.416 & 0.253 & 0.014 & 0.068 & -0.099 & 0.110 & -0.021 & 0.031 \\
\hline$i_{0}$ & -0.089 & -0.083 & -0.124 & 0.192 & -0.416 & 1.000 & -0.979 & 0.073 & -0.099 & 0.103 & -0.167 & 0.083 & -0.095 \\
\hline$d i / d r$ & 0.108 & 0.102 & 0.119 & -0.167 & 0.253 & -0.979 & 1.000 & -0.075 & 0.090 & -0.088 & 0.147 & -0.086 & 0.097 \\
\hline$d^{2} \Omega / 2 d r^{2}$ & 0.089 & 0.095 & -0.239 & 0.045 & -0.099 & 0.103 & -0.088 & 0.972 & -0.995 & 1.000 & 0.028 & 0.211 & -0.19 \\
\hline 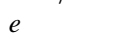 & -0.060 & -0.058 & 0.051 & -0.695 & 0.110 & -0.167 & 0.147 & 0.047 & -0.037 & 0.028 & 1.000 & 0.074 & 0.01 \\
\hline$\omega_{0}$ & 0.301 & 0.311 & -0.413 & -0.241 & -0.021 & 0.083 & -0.086 & 0.211 & -0.214 & 0.211 & 0.074 & 1.000 & -0.97 \\
\hline$d \omega / d r$ & -0.285 & -0.298 & 0.423 & 0.159 & 0.031 & -0.095 & 0.097 & -0.199 & 0.200 & -0.197 & 0.015 & -0.979 & 1.00 \\
\hline
\end{tabular}
solution for distance with $D=W^{1 / 3} s^{4 / 3} a^{-1}$.

Table 6

Parameter Correlation Matrix (See Section 5.3) 


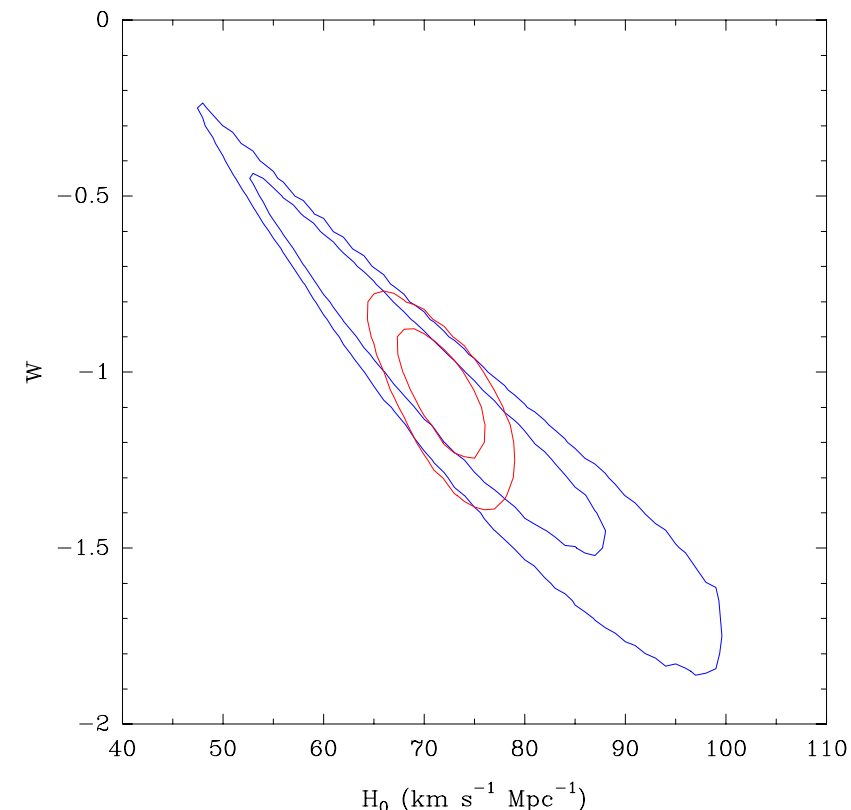

Figure 8. Constraint on dark energy. 2D probability density functions (PDFs) for a constant equation of state of dark energy $(w)$ and $H_{0}$ with $95 \%$ and $68 \%$ confidence contours. The blue contours were generated by binning the parameter values from Markov chains from the WMAP $7 \mathrm{yr}$ data (modeled with a constant $w, \Lambda \mathrm{CDM}$ model that incorporates the effects of the SZ effect and gravitational lensing). The red contours combine the WMAP PDF and the constraint that $H_{0}=72.0 \pm 3.0 \mathrm{~km} \mathrm{~s}^{-1} \mathrm{Mpc}^{-1}$ from the results presented in this paper. Fitting a Gaussian to the marginalized one-dimensional PDF for $w$ yields $w=-1.06 \pm 0.12$ ( $\pm 68 \%$ confidence).

(A color version of this figure is available in the online journal.)

However, $a, s$, and $W$ are statistical quantities. If $W$ is known rather accurately, as in our data set, then a narrow ellipse of "allowed" values of $M_{\mathrm{bh}}$ and $D$ forms in $M_{\mathrm{bh}}-D$ space (Figure 5), the size of which is determined by the $1 \sigma$ uncertainties in $a, s$, and $W$. Outside of the ellipse, solutions are not probable as the intersecting curves separate. The $M_{\mathrm{bh}}-D$ joint probability distribution is highly elliptical, which explains the high correlation coefficient (Kuo et al. 2013).

In summary, the distance to the NGC 4258 disk can be reliably determined. Possible solutions are constrained to lie within an ellipse in the $M_{\mathrm{bh}}-D$ space, the size of which is governed by the $1 \sigma$ uncertainties in $a, s$, and $W$.

Note that there are other high correlations in Table 6. However, these are caused by defining the disk warping terms relative to $r=0$. Had they been defined relative to $r=r_{\text {mid }}$, where $r_{\text {mid }}$ is the mid-radius of maser features in the disk, then they would be small. This has no effect on the distance estimate.

\subsection{Maser Distance to NGC 4258}

Adding all of the systematic uncertainty terms in Table 4 yields a distance of $7.60 \pm 0.17$ (random) \pm 0.15 (systematic) Mpc. Combining the random and systematic uncertainties in the quadrature gives a $\pm 3 \%$ distance uncertainty. This is consistent with, but considerably better than, the result of H99.

\section{IMPLICATIONS OF THE DISTANCE FOR $H_{0}$}

As noted in Section 1, the maser distance to NGC 4258 cannot be used to directly calculate a high-accuracy $H_{0}$, since it is relatively nearby and its peculiar motion could be a large proportion of its recessional velocity. Instead, the role for NGC 4258 is to be an anchor for the Cepheid-calibrated extragalactic distance scale. Using a maser distance to NGC 4258 of $7.28 \mathrm{Mpc} \pm 3 \%$ (Riess et al. 2012), Riess et al. (2011) obtained $H_{0,4258}=74.8 \pm$ $3.1 \mathrm{~km} \mathrm{~s}^{-1} \mathrm{Mpc}^{-1}$. We can now revise this value for the Hubble constant to $H_{0,4258}=72.0 \pm 3.0 \mathrm{~km} \mathrm{~s}^{-1} \mathrm{Mpc}^{-1}$.

This is in good agreement with the $H_{0}$ estimated by the sevenyear Wilkinson Microwave Anistropy Probe (WMAP) data of $71.0 \pm 2.5 \mathrm{~km} \mathrm{~s}^{-1} \mathrm{Mpc}^{-1}$ for standard $\Lambda$-CDM cosmology and a flat universe (Larson et al. 2011), and that for WMAP + Baryonic Acoustic Oscillations of $69.3 \pm 0.9 \mathrm{~km} \mathrm{~s}^{-1} \mathrm{Mpc}^{-1}$ (Hinshaw et al. 2012), and in less good agreement (a $1.5 \sigma$ discrepancy) with $H_{0}$ from the Planck satellite of $67.3 \pm 1.2 \mathrm{~km} \mathrm{~s}^{-1} \mathrm{Mpc}^{-1}$ (Planck Collaboration et al. 2013). The maser cosmology project has reported two estimates of $H_{0}$ based on the same procedures for estimating distance as that used here (i.e., maser distribution plus accelerations) with the results: UGC 3789 (distance = 49.6 Mpc) $H_{0}=68.7 \pm 7.1 \mathrm{~km} \mathrm{~s}^{-1} \mathrm{Mpc}^{-1}$ (Reid et al. 2013) and NGC 6264 (distance $=144 \pm 19 \mathrm{Mpc}$ ) $H_{0}=68 \pm$ $9 \mathrm{~km} \mathrm{~s}^{-1} \mathrm{Mpc}^{-1}$ (Kuo et al. 2013). These masers are far enough away that $H_{0}$ could be calculated with negligible error due to the peculiar motions of the galaxies. The weighted mean estimate of $H_{0}$ for these two galaxies is $68.7 \pm 5.6 \mathrm{~km} \mathrm{~s}^{-1} \mathrm{Mpc}^{-1}$.

\section{CONCLUSIONS}

We analyzed 18 epochs of VLBI water maser data and more than $10 \mathrm{yr}$ of acceleration monitoring in Papers I and II. Here, we fitted the resulting data set using a Bayesian method to yield a new, high-accuracy, and purely geometric maser distance to NGC 4258. We took particular care to assess terms of systematic uncertainty and obtained a distance of $7.60 \pm 0.23 \mathrm{Mpc}$ (i.e., $\pm 3 \%$ ), which is consistent with, but much more accurate than, the H99 maser distance estimate of $7.2 \pm 0.5 \mathrm{Mpc}(7 \%)$. The new distance estimate yields an $H_{0,4258}=72.0 \pm 3.0 \mathrm{~km} \mathrm{~s}^{-1} \mathrm{Mpc}^{-1}$ which provides an important independent estimate of the Hubble constant. The Planck Collaboration et al. (2013) commented on the "tension" between their result and the "local" value of $H_{0}$ derived from Cepheid measurements. We note that our result lies between these. The use of water masers to derive the Hubble constant remains important because it does not share the systematic uncertainties of the other methods, e.g., the $\Lambda$-CDM model parameters in the case of the cosmic microwave background derived Hubble constant and the distance ladder assumptions associated with the Cepheid method. The determination of $H_{0}$ is approaching the level required to impose additional constraints on the equation-of-state parameter for dark energy (Weinberg et al. 2012, see Figure 8).

We thank the anonymous referee, Adam Riess, and Lucas Macri for comments that improved the manuscript. We also thank Carolann Barrett for proofreading the manuscript.

Facilities: VLBA, VLA, GBT

\section{APPENDIX A}

\section{COORDINATE SYSTEMS}

Consider a maser disk plane coordinate system $(x, y, z)$ and a sky-plane system denoted by $(X, Y, Z)$ with a supermassive black hole at the common origin of both systems (Figure 9).

Drawing on the notation of Peiris \& Tremaine (2003), the sky-plane system has the $(X, Y)$ plane as the sky plane with positive $X$ pointing east and positive $Y$ pointing north, a right-handed coordinate system. The positive $Z$-axis points 


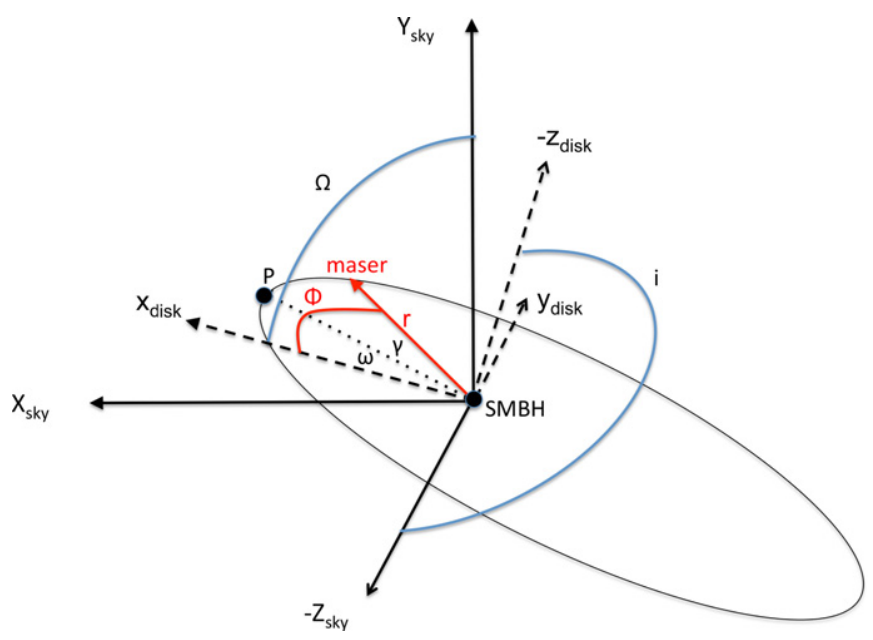

Figure 9. Geometry of the maser disk-sky plane coordinate systems. $X_{\text {sky }}-Y_{\text {sky }}$ is the sky plane and $x_{\text {disk }}-y_{\text {disk }}$ is the maser disk plane. The supermassive black hole $(\mathrm{SMBH})$ marks the zero point of both coordinate systems. $-z_{\text {disk }}$ marks the direction of the negative angular momentum for the maser disk, and the angle between $-z_{\text {disk }}$ and $-Z_{\text {sky }}$, which is the direction to the observer, is the inclination angle $i$. The perihelion of the maser orbit displayed is marked with a $P$. The position of maser features in the disk is measured from the disk $x$-axis, which is confined to lie in the sky plane, and is the angle $\phi$ for each feature. The periapsis angle is the angle $\omega$ and the disk position angle, $\Omega$, is measured in the sky plane in the direction east of north to the disk $x$-axis.

(A color version of this figure is available in the online journal.)

along the LOS away from the observer, and the LOS velocity $v_{\text {los }}=\dot{Z}$ is positive for receding objects. The second system is the disk-plane system in which coordinates of the disk plane are denoted as $(x, y, z)$. The $(x, y)$ plane is in the plane of the disk with the positive $x$-axis pointing along the sky $X$-axis. The positive $z$-axis points in the direction of the disk angular momentum vector (i.e., also a right-handed coordinate system).

To convert between sky $(X, Y, Z)$ and disk $(x, y, z)$ coordinate frames for a flat, inclined maser disk, we perform rotations about two Euler angles $(\Omega, i)$, where $\Omega$ is the angle to the disk $x$-axis measured east of north (the $Y$-axis) in the $(X, Y)$ plane and is the disk position angle and $i$ is the inclination angle measured between $-Z$ and $-z$. The first rotation is by $i$ about the sky $X$-axis and the second rotation is by $\Omega$ about the sky $Z$-axis, i.e.,

$$
\begin{aligned}
\left(\begin{array}{l}
X \\
Y \\
Z
\end{array}\right)= & \left(\begin{array}{ccc}
\sin \Omega & -\cos \Omega & 0 \\
\cos \Omega & \sin \Omega & 0 \\
0 & 0 & 1
\end{array}\right) \\
& \times\left(\begin{array}{ccc}
1 & 0 & 0 \\
0 & \cos i & -\sin i \\
0 & \sin i & \cos i
\end{array}\right)\left(\begin{array}{l}
x \\
y \\
z
\end{array}\right)
\end{aligned}
$$

where the coordinates of a maser feature in the disk frame are $(x, y, z)=(r \cos \phi, r \sin \phi, 0)$.

The coordinates of masers in the sky reference frame are therefore given by

$$
\begin{aligned}
& X=r(\sin \Omega \cos \phi-\cos \Omega \cos i \sin \phi) \\
& Y=r(\cos \Omega \cos \phi+\sin \Omega \cos i \sin \phi) \\
& Z=r \sin i \sin \phi .
\end{aligned}
$$

\section{APPENDIX B}

\section{VELOCITY AND ACCELERATION}

For defining velocity, the angle of the maser in the disk with respect to perihelion, periapsis angle $\omega$, now becomes relevant i.e., $\gamma=(\phi-\omega)$. Velocity in disk polar coordinates is given by $v=\dot{r}+r \dot{\gamma}$, where $v_{r}=\dot{r}$ and $v_{\gamma}=r \dot{\gamma}$ are radial and tangential velocity components, respectively. In this Appendix, $r$ is a linear radius rather than an angular one. Specific angular momentum $h$ is given by $h=2 \pi a b / P$, where $a$ and $b$ are the semi-major and minor axes, respectively, and $P$ is the orbital period. As $b=$ $a \sqrt{\left(1-e^{2}\right)}$ and $P^{2}=4 \pi^{2} a^{3} / G M, h=\sqrt{a\left(1-e^{2}\right) G M}$. Since $\dot{\gamma}=h / r^{2}$ and $r=a\left(1-e^{2}\right) /(1+e \cos \gamma), v_{\gamma}=r \dot{\gamma}=r h / r^{2}=$ $\sqrt{a\left(1-e^{2}\right) G M r^{2} / r^{4}}=\sqrt{G M(1+e \cos \gamma) / r}$, and $v_{r}=\dot{r}=$ $\left.a\left(1-e^{2}\right) /(1+e \cos \gamma)^{2}\right) e \sin \phi \dot{\gamma}=\sqrt{G M / r(1+e \cos \gamma)} e \sin \gamma$.

Velocity components in the disk frame are denoted by $\left(v_{x}, v_{y}, v_{z}\right)=\left(v_{r} \cos \phi-v_{\gamma} \sin \phi, v_{r} \sin \phi+v_{\gamma} \cos \phi, 0\right)$ and in the sky frame by

$$
\begin{aligned}
v_{X}= & v_{r}(\sin \alpha \cos \phi-\cos \alpha \cos i \sin \phi) \\
& +v_{\gamma}(\cos \alpha \cos i \cos \phi-\sin \alpha \sin \phi) \\
v_{Y}= & v_{r}(\cos \alpha \cos \phi+\sin \alpha \cos i \sin \phi) \\
& +v_{\gamma}(\sin \alpha \cos i \cos \phi-\cos \alpha \sin \phi) \\
v_{Z}= & v_{r} \sin i \sin \phi+v_{\gamma} \sin i \cos \phi .
\end{aligned}
$$

Acceleration is given by $a=a_{r}+a_{\gamma}=\ddot{r}-r(\dot{\gamma})^{2}+r \ddot{\gamma}+2 \dot{r} \dot{\gamma}$, where the centripetal acceleration component $a_{r}=-G M r^{-2}$ and $a_{\gamma}=0$. In the disk frame, the components of $a_{r}$ are $\left(a_{x}, a_{y}, a_{z}\right)=\left(-a_{r} \cos \phi,-a_{r} \sin \phi, 0\right)$, so that in the LOS to the observer, $a_{Z}=-a_{r} \cos i \sin i \sin \phi-a_{r} \sin \phi \sin i \cos \phi=$ $-G M r^{-2} \sin i \sin \phi$.

\section{APPENDIX C}

\section{RELATIVISTIC EFFECTS}

When modeling water maser emission from AGN disks, one must relate a model velocity to the observed (e.g., optical or radio-definition) velocities.

Starting in a reference frame located at the focus of the maser orbits (the black hole frame), gas clouds at radius, $r$, orbit with velocity, $v_{\text {orb }}=\sqrt{G M / r D}$, where $M$ is the mass of the black hole (and providing one is not near the strong gravity regime). Our observing frame is receding from the black hole frame at velocity $v_{\text {rec }}$.

We will use the relativistic Doppler equation (see Rybicki \& Lightman 1986, p. 111, Equation (4.11)), which gives the relation between frequency, $f$, in the source rest frame to the frequency, $F$, in an observer's frame moving with velocity $v$ relative to the source:

$$
F=f \Gamma^{-1} /\left(1-\frac{v}{c} \cos \theta\right),
$$

where $\theta$ is the angle between the vector $v$ and our LOS and

$$
\Gamma=1 / \sqrt{1-\left(v^{2} / c^{2}\right)}
$$

(Note that $v$ is implicitly $\geqslant 0$ and $\cos \theta$ can be positive or negative, i.e., coming toward or away from the observer, respectively).

In a frame at rest with a masing cloud, the emission is at the rest frequency $f_{0}$. Such a cloud is moving with respect to the black hole frame by its orbital velocity, $v_{\text {orb}}$, which can be decomposed into parallel (toward the observer) and perpendicular components, $v_{\|}$and $\mathrm{v}_{\perp}$. Imagine observing in this frame at the distance of the Sun, but still at rest in the black hole frame (i.e., zero recessional velocity). Using the relativistic 
Doppler equation,

$$
f=f_{0} \Gamma^{-1} /\left(1+\frac{v_{\|}}{c}\right)
$$

Next, transform from the black hole frame to the observers' frame, which is receding at velocity $v_{\text {rec }}$ along the LOS. Again using the relativistic Doppler equation,

$$
F=f \Gamma^{-1} /\left(1+\frac{v_{\mathrm{rec}}}{c}\right) .
$$

$F$ gives the observed frequency accounting for all special relativistic effects, including time dilation and light-travel effects. However, as the photons travel away from the black hole, they experience a general relativistic "gravitational redshift." This reduces the observed frequency further such that the observed frequency is given by

$$
F_{\mathrm{gr}}=F \sqrt{1-R_{\mathrm{sch}} / r},
$$

where $R_{\text {sch }}=2 G M / c^{2}$.

Finally, if, for example, the observational data we seek to model use the optical definition of velocity,

$$
v_{\mathrm{los}}^{\prime} \equiv c\left(\frac{f_{0}}{F_{\mathrm{gr}}}-1\right)
$$

or equivalent conversions for other velocity definitions can be made.

\section{REFERENCES}

Argon, A. L., Greenhill, L. J., Reid, M. J., Moran, J. M., \& Humphreys, E. M. L. 2007, ApJ, 659, 1040
Armitage, P. J. 2008, arXiv:0802.1524

Cecil, G., Wilson, A. S., \& Tully, R. B. 1992, ApJ, 390, 365

Freedman, W. L., Madore, B. F., Gibson, B. K., et al. 2001, ApJ, 553, 47

Greenhill, L. J., Henkel, C., Becker, R., Wilson, T. L., \& Wouterloot, J. G. A. 1995, A\&A, 304, 21

Herrnstein, J. R., Moran, J. M., Greenhill, L. J., \& Trotter, A. S. 2005, ApJ, 629,719

Herrnstein, J. R., Moran, J. M., Greenhill, L. J., et al. 1997, ApJL, 475, L17

Herrnstein, J. R., Moran, J. M., Greenhill, L. J., et al. 1999, Natur, 400,539

Hinshaw, G., Larson, D., Komatsu, E., et al. 2012, arXiv:1212.5226

Humphreys, E. M. L., Argon, A. L., Greenhill, L. J., Moran, J. M., \& Reid, M. J. 2005, in ASP Conf. Ser. 340, Future Directions in High Resolution Astronomy, ed. J. Romney \& M. Reid (San Francisco, CA: ASP), 466

Humphreys, E. M. L., Reid, M. J., Greenhill, L. J., Moran, J. M., \& Argon, A. L. 2008, ApJ, 672, 800

Kuo, C. Y., Braatz, J. A., Reid, M. J., et al. 2013, ApJ, 767, 155

Larson, D., Dunkley, J., Hinshaw, G., et al. 2011, ApJS, 192, 16

Macri, L. M., Stanek, K. Z., Bersier, D., Greenhill, L. J., \& Reid, M. J. 2006, ApJ, 652,1133

Maoz, E. 1995, ApJL, 455, L131

Moran, J. M., Humphreys, E., Greenhill, L., Reid, M., \& Argon, A. 2007, in in IAU Symp. 242, Astrophysical Masers and Their Environments, ed. J. M. Chapman \& W. A. Baan (Cambridge: Cambridge Univ. Press), 391

Peiris, H. V., \& Tremaine, S. 2003, ApJ, 599, 237

Planck Collaboration Ade, P. A. R., Aghanim, N., et al. 2013, arXiv:1303.5076

Reid, M. J., Braatz, J. A., Condon, J. J., et al. 2013, ApJ, 767,154

Riess, A. G., Macri, L., Casertano, S., et al. 2011, ApJ, 730, 119

Riess, A. G., Macri, L., Casertano, S., et al. 2012, ApJ, 752, 76

Rybicki, G. B., \& Lightman, A. P. 1986, Radiative Processes in Astrophysics (New York: Wiley)

Statler, T. S. 2001, AJ, 122, 2257

Statler, T. S. 2002, in ASP Conf. Ser. 275, Disks of Galaxies: Kinematics, Dynamics and Peturbations, ed. E. Athanassoula, A. Bosma, \& R. Mujica (San Francisco, CA: ASP), 276

Weinberg, D. H., Mortonson, M. J., Eisenstein, D. J., et al. 2012, arXiv:1201.2434 